\title{
Effect of endoplasmic reticulum stress involved in manganese-induced neurotoxicity in rats
}

\author{
CAILIAN WU ${ }^{1 *}$, GUANDOU YUAN ${ }^{2 *}$, RUIKANG MO ${ }^{3}$, YANNING HUANG $^{4}$, \\ TIANTIAN LUO ${ }^{1}$ and JIN WANG ${ }^{4}$ \\ ${ }^{1}$ Mental Health Center, The Second Affiliated Hospital of Guangxi Medical University, Nanning, Guangxi 530007; \\ ${ }^{2}$ Department of Hepatobiliary Surgery, The First Affiliated Hospital of Guangxi Medical University, \\ Nanning, Guangxi 530021; ${ }^{3}$ Department of Neurology, The First People's Hospital of Nanning, \\ Nanning, Guangxi 530022; ${ }^{4}$ Department of Neurology, The First Affiliated Hospital of \\ Guangxi Medical University, Nanning, Guangxi 530021, P.R. China
}

Received May 17, 2018; Accepted December 31, 2018

DOI: $10.3892 / \mathrm{mmr} .2019 .10175$

\begin{abstract}
The aim of the present study was to probe the mechanism of apoptosis induced by endoplasmic reticulum stress (ERS) in manganese-induced rats. A total of 60 Sprague-Dawley rats were randomly divided into a Vehicle group, LoMag group, HiMag group, and HiMag + 4-phenylbutyrate (PBA) group. Manganese content was measured by Inductively Coupled Plasma-Atomic Emission Spectrometry. Pathogenic morphology, the cellular structure of the striatum and ER were observed by hematoxylin and eosin staining and electron microscopy. The TUNEL method was used to examine neuronal apoptosis in the rat striatum. The expression levels of glucose-regulated protein 78KD (GRP78), C/EBP homologous protein (CHOP), c-Jun N-terminal kinase (JNK) and caspase- 12 were analyzed by western blot analysis. The results revealed that striatal manganese concentrations in the LoMag and HiMag groups were higher than that in the Vehicle group $(\mathrm{P}<0.01)$. Rat striatal neuronal structure and apoptotic rates in the LoMag and HiMag groups were higher than those in the Vehicle group $(\mathrm{P}<0.05)$. 4-PBA treatment effectively reduced the apoptotic cell number $(\mathrm{P}<0.05)$. In addition, ER swelling and vacuolization in the HiMag + PBA group was reduced compared with that in the HiMag group. In addition, the protein expression levels of GRP78, CHOP, JNK and caspase-12 in the LoMag and HiMag groups were higher than those in the Vehicle group $(\mathrm{P}<0.05)$. However, the expression of these four proteins was reduced by 4 -PBA treatment $(\mathrm{P}<0.05)$.
\end{abstract}

Correspondence to: Dr Jin Wang, Department of Neurology, The First Affiliated Hospital of Guangxi Medical University, 6 Shuangyong Road, Nanning, Guangxi 530021, P.R. China

E-mail: j_wang18@outlook.com

*Contributed equally

Key words: rat, manganese, endoplasmic reticulum stress, neurotoxicity
In conclusion, 4-PBA significantly reduced the damage and apoptosis induced by manganese exposure in rats.

\section{Introduction}

Manganese, as a rich metal element in the earth, is widely found in nature (1). Manganese has been shown to be a necessary factor for various enzymes in humans, including manganese superoxide dismutase and pyruvate carboxylase, which are involved in protein synthesis, neurotransmitter transmission and nervous system development. Manganese enters the human body primarily through respiration. Long-term and high-dose exposure to manganese can lead to various types of damage to human organs. The brain is the major organ which can be damaged by manganese exposure. In addition, manganese can pass through the blood-brain barrier and accumulate in the brain basal ganglia, striatum and substantia nigra $(2,3)$. The mechanisms of manganese-induced neurological damage have been described to involve dopamine oxidative damage, oxygen free radicals, excitotoxicity, functional imbalances in mitochondria and iron metabolism disorders. However, the detailed molecular mechanism remains to be fully elucidated (4). It has been shown that endoplasmic reticulum stress (ERS) may be involved in the pathogenesis of neurotoxicity caused by manganese poisoning. The endoplasmic reticulum (ER) is a subcellular structure, which is involved in protein synthesis, processing and transport in cells. The ER is key in maintaining intracellular calcium stabilization (5). An absence of energy, calcium homeostasis, and oxidative stress conditions destroy the normal ER physiological function, resulting in the misfolding of proteins. Subsequently, these adverse reactions can induce ERS and the unfolding protein response (UPR).

Glucose regulated protein 78KD (GRP78) is an ER chaperone protein. GRP78 has been shown to assist in the processing and modification of new synthetic proteins. Therefore, GRP78 is considered to be a landmark protein for detecting ERS (6). Under a non-stressed state, three transmembrane receptors, including double-stranded RNA-dependent protein kinase-like ER kinase (PERK), activation of transcription factor 6 (ATF6) and type I transmembrane protein kinase (IRE-1), can bind to 
GRP78 and maintain an inactive state of GRP78. When ERS occurs, a large number of abnormal proteins are produced. These abnormal protein molecules occupy the binding sites on GRP78, which cause the dissociation of IRE-1, PERK and ATF6 from GRP78. In the initial stage of ERS, the activation of chaperones promotes degradation of the incorrect proteins and restores cell homeostasis. However, if the stress exceeds the ability of the cells to maintain their homeostasis, PERK, ATF6 and IRE- $\alpha$ activate the apoptotic program to degenerate cells. Previous studies have suggested that C/EBP homologous protein (CHOP), c-Jun N-terminal kinase (JNK) and caspase-12 are key in ERS-activated apoptosis: i) Activation of $\mathrm{CHOP} /$ growth arrest and DNA damage-inducible 153 gene transcription through the PERK/eukaryotic translation initiation factor $2 \alpha$ (eIF $2 \alpha$ ) pathway $(7,8)$. This signaling pathway can reduce anti-apoptotic gene B-cell lymphoma 2 (Bcl-2) translation, and upregulate the expression of pro-apoptotic factor Bcl-2-assocated X protein (Bax); this accelerates cell apoptosis; ii) IRE1 can activate the JNK signaling pathway, which downregulates the expression of $\mathrm{Bcl}-2$. The low expression of $\mathrm{Bcl}-2$ upregulates the gene expression of pro-apoptotic Bcl-2-interacting mediator of cell death and mitochondrial cascade activation (9); iii) ERS can specifically activate caspase-12, which is located in the ER. Caspase-12 can regulate the expression of apoptotic promoters caspase-9 and caspase-3, which ultimately leads to cell function impairment (10). ERS inhibitor 4-phenylbutyrate (4-PBA), as a chaperone, can stabilize the protein structure and transports large quantities of aggregated proteins in the ER. Therefore, 4-PBA can reduce the ER burden and inhibit the occurrence of ERS. It has been identified that 4-PBA can inhibit ERS. For example, reducing blood glucose levels in patients with diabetes (11), improving the insulin secretion barrier (12), alleviating the occurrence of diabetic nephropathy (13), reducing ischemia in liver cells (14), and reducing nerve cell apoptosis and hypoxic ischemic injury $(15,16)$.

In the present study, a sub-acute manganese exposure rat model was established by intraperitoneal injection of manganese. Hematoxylin and eosin (H\&E) staining, a TUNEL assay and electron microscopy were used to evaluate the effect of manganese on neurotoxicity. Western blot analysis was used to determine whether ERS was involved in the neurotoxicity. In addition, the potential value of 4-PBA in neuron protection was probed. The results of the study provide a basis for the early diagnosis and treatment of manganese affecting a population.

\section{Materials and methods}

Experimental animals and groups. The experiment was approved by the Committee on the Ethics of Animal Experiments of the First Affiliated Hospital of Guangxi Medical University [Nanning, China; permit no. 2015 (KY-E-67)]. A total of 60 healthy male Sprague-Dawley rats, aged 3-4 months old and weighing $200 \pm 20 \mathrm{~g}$, were obtained from the Animal Center of Guangxi Medical University (certificate of conformity no. SCXK Gui 2014 0002). The animal room was maintained in clean and sterile environment, with a relative humidity of $40-50 \%$, and $22-25^{\circ} \mathrm{C}$ room temperature. The cage was paved with cleaning accessories. The rats were free to move within the cage, which was equipped with black holes to avoid danger. The experimental animals were healthy, disease-free and had normal physiological functions. All rats were housed in quiet conditions to avoid fright or panic. The rats were provided with clean feed and free water. Following 3 days of adaptive feeding, the rats were randomized divided into four subgroups using a random number table: i) Vehicle group ( $n=15)$; ii) LoMag group ( $n=15)$; iii) HiMag group $(\mathrm{n}=15)$; and iv) HiMag + PBA group $(\mathrm{n}=15)$. The animal model was constructed according to previous studies $(15,17)$. The rats in the four groups were injected intraperitoneally with the same volume of normal saline. The rats in the LoMag group and HiMag group were administered with an intraperitoneal injection of 6 and $15 \mathrm{mg} / \mathrm{kg} \mathrm{MnCl}{ }_{2}$ (Sigma-Aldrich; Merck KGaA, Darmstadt, Germany), respectively. The rats in the HiMag + PBA group were administered with an intraperitoneal injection of $200 \mathrm{mg} / \mathrm{kg}$ 4-PBA (Sigma-Aldrich; Merck KGaA) and, after $30 \mathrm{~min}, 15 \mathrm{mg} / \mathrm{kg} \mathrm{MnCl}_{2}$ was administered by intraperitoneal injection. The injection volume of each solution was calculated using the following formula: Injection volume $=$ rat weight $/(2 \mathrm{ml} / \mathrm{kg})$. The rats were injected daily between 6:00 and 7:00 pm and injected weekly for 6 days. The health status and behavioral changes of the animals were observed every day. In addition, the following criteria were applied to determine when the animals should be sacrificed in advance to avoid causing pain to the animal: Abdominal infection, weight loss, loss of appetite and weakness. After 4 weeks of continuous injection, five rats were randomly selected from each group. The rats were anesthetized and decapitated $24 \mathrm{~h}$ following the final $\mathrm{MnCl}_{2}$ injection. All rats were anesthetized through intraperitoneal injection of $300 \mathrm{mg} / \mathrm{kg}$ body weight of $10 \%$ (w/v) chloral hydrate solution (National Drug Approval no. H37022673, Qingdao Yulong Algae Co., Ltd., Qingdao, China). The anesthetized rats were then placed under a heating light to maintain normal body temperature. Subsequently, the hearts and brains were separated immediately following anesthesia. The heart was perfused with $0.9 \%$ frozen saline ( $250 \mathrm{ml}$; Sigma-Aldrich; Merck KGaA). The brain striatum was stored in a $-80^{\circ} \mathrm{C}$ cryogenic refrigerator for further analysis.

Striatal manganese content determination. The same quantity of rat striatum tissue was weighed accurately using an electronic balance (Thermo Fisher Scientific, Inc., Waltham, MA, USA), and then transferred into $10-\mathrm{ml}$ tubes. Subsequently, $3 \mathrm{ml}$ mixed acid (concentrated $\mathrm{HNO}_{3}$, concentrated $\mathrm{H}_{2} \mathrm{SO}_{4}$ and concentrated $\mathrm{HCI}$ ) prepared at a ratio of 9: 1: 1 (Sigma-Aldrich; Merck KGaA) was added into the 10 -ml tube to digest tissues at $37^{\circ} \mathrm{C}$ overnight. The following day, the solution was digested and clarified to near dryness in an adjustable electric furnace. Ultra-pure water was added to $5 \mathrm{ml}$. The $\mathrm{Mn}$ content was measured by ICP-AES with default parameters (PerkinElmer, Inc., Waltham, MA, USA).

Sectioning. Five rats were randomly selected from each group. Following anesthesia, thoracotomy was performed and the apical catheter was inserted into the ascending aorta. The right atrial appendage was cut off, and $250 \mathrm{ml}$ of $0.9 \%$ frozen normal saline (Sigma-Aldrich; Merck KGaA) was rapidly infused. Subsequently, $200 \mathrm{ml}$ of $4 \%$ paraformaldehyde 
solution (Sigma-Aldrich; Merck KGaA) was infused. The brain striatum was rapidly removed, and the tissue was placed in $4 \%$ paraformaldehyde (Sigma-Aldrich; Merck KGaA) for $8 \mathrm{~h}$. The specimens were diced and placed in $75,85,95$, and $100 \%$ gradient in ethanol (Sigma-Aldrich; Merck KGaA) for dehydration. Xylene-soaked transparent processing was performed prior to wax embedding. The slice thickness was $\sim 5 \mu \mathrm{m}$. All slices were fixed at $60^{\circ} \mathrm{C}$ for $3 \mathrm{~h}$.

$H \& E$ and TUNEL staining. The H\&E staining kit (Beyotime Institute of Biotechnology, Haimen, China) and TUNEL assay (Beyotime Institute of Biotechnology) were used to detect neuronal apoptosis in the striatum. H\&E staining was performed with the following steps: Xylene dewaxing for $15 \mathrm{~min}$, xylene washed with gradient alcohol, hematoxylin dye staining $15 \mathrm{~min}$ after distilled water washing for $5 \mathrm{~min}$, hydrochloric acid color separation for $20 \mathrm{sec}$, distilled water immersion for $10 \mathrm{~min}, 0.5 \%$ eosin soak $20 \mathrm{sec}$ after washing, gland packing sheet after natural drying, sample observation using a light microscope (magnification, $\mathrm{x} 200$ or $\mathrm{x} 400$ ). The TUNEL method was used to detect striatal neuron apoptosis. The detailed steps were as follows: Conventional paraffin section, dewaxing, washing in PBS for $5 \mathrm{~min}, 3 \%$ hydrogen peroxide incubation at $37^{\circ} \mathrm{C}$ for $20 \mathrm{~min}$, washing in PBS for $5 \mathrm{~min}$ (repeat once), addition of marker solution and incubation at $37^{\circ} \mathrm{C}$ in the dark for $2 \mathrm{~h}$, washing in PBS for $5 \mathrm{~min}$, addition of POD reagent and incubation at $37^{\circ} \mathrm{C}$ for $30 \mathrm{~min}$, washing in PBS, drying, DAB colorization, drying, placement in a neutral gland plate, sample observation using a light microscope (magnification, $\mathrm{x} 400$ ). The neuronal apoptotic index (AI) was calculated as follows: $\mathrm{AI}=$ nuclei of apoptotic cells/nuclei of total cells x $100 \%$.

Transmission electron microscopy. In the present study, transmission electron microscopy (FEI; Thermo Fisher Scientific, Inc.) was used to observe stromal cell structure changes and ER morphology. Five rats in each group were randomly selected. The brain specimens were rinsed with PBS (Sigma-Aldrich; Merck KGaA) and fixed with $1 \%$ osmium tetroxide solution (Sigma-Aldrich; Merck KGaA). Subsequently, the treated specimens were dehydrated with gradient alcohol and treated with acetone and embedding agent overnight. The embedded samples were sectioned ( $65-80 \mathrm{~nm}$ in thickness). The sections were stained with lead citrate for $15 \mathrm{~min}$ and stained with $50 \%$ ethanolic uranyl acetate (Sigma-Aldrich; Merck KGaA) for $15 \mathrm{~min}$. The sample was observed under a transmission electron microscope.

Western blot analysis. Western blot analysis was used to detect changes in the expression of ERS-related proteins (GRP78, CHOP, JNK and caspase-12). The tissue was transferred into a 1.5-ml EP tube. Eye scissors were used to cut the samples into pieces on ice, and pre-cooled cell lysate was added (mixture of PMSF and RIPA cell lysate at a volume ratio of 1:100; Sigma-Aldrich; Merck KGaA) to $0.5 \mathrm{ml}$. The sample was ground evenly until no tissue residue was present. The slurry was placed in a low-temperature high-speed centrifuge (Eppendorf, Hamburg, Germany), and centrifuged at $9,500 \times \mathrm{g}$ at $4^{\circ} \mathrm{C}$ for $5 \mathrm{~min}$. The supernatant was transferred into a new EP tube and maintained at $-80^{\circ} \mathrm{C}$. The protein concentration was quantified using the bicinchoninic acid assay method. Protein concentration was detected at $562 \mathrm{~nm}$ using the Varioskan ${ }^{\mathrm{TM}}$ LUX multimode reader (Thermo Fisher Scientific, Inc.). The protein standard curve was calculated prior to the calculation of the protein concentration in each sample. Following boiling with SDS-PAGE sample buffer (Sigma-Aldrich; Merck KGaA) for $5 \mathrm{~min}, 100 \mu \mathrm{g}$ total protein was loaded in each lane. Proteins were separated by $10 \%$ SDS-PAGE. The proteins were then transferred onto a polyvinylidene difluoride membrane (EMD Millipore, Billerica, MA, USA). Following blocking with western blot blocking buffer (cat. no. T7132A; Takara Bio, Inc., Otsu, Japan) for $1 \mathrm{~h}$ at room temperature, the membrane was incubated with a 1:1,000 diluted primary antibody at $4^{\circ} \mathrm{C}$ overnight. The primary antibodies used were the following: Rabbit polyclonal anti-mouse GRP78 (cat. no. M00955), CHOP (cat. no. M00311), JNK (cat. no. M02608) caspase-12 (cat. no. A04700) and $\beta$-actin (cat. no. M01263). All primary antibodies were purchased from Boster Biological Technology (Pleasanton, CA, USA). Prior to detection with an ECL chemiluminescence detection kit (Beyotime Institute of Biotechnology), the proteins were incubated with the corresponding secondary antibody (cat. no. BA1001; dilution, $1: 5,000)$ for $1 \mathrm{~h}$ at room temperature (Boster Biological Technology). The bands were examined using an Odyssey Infrared Imaging system (LI-COR Biosciences, Lincoln, NE, USA) scan; the resolution of the instrument ranges between 21 and $339 \mu \mathrm{m}$, and images of the blots and gels were captured at $169 \mu \mathrm{m}$. Quantification was performed on single channels with the Odyssey CLx software (version 1.0.9; LI-COR Biosciences) as previously described (18).

Statistical analysis. SPSS 17.0 (SPSS, Inc., Chicago, IL, USA) was used for statistical analysis. Data for manganese content, neuron apoptotic rate, protein expression and other data are expressed as the mean \pm standard deviation. One-way analysis of variance was applied. If there were differences in the overall data, further comparison of individual data was performed. The LSQ test was applied if variance was the same. Tamhane's T2 method was used if variance was not the same. $\mathrm{P}<0.05$ was considered to indicate a statistically significant difference.

\section{Results}

4-PBA has no effect on manganese content in the rat striatum. In order to examine whether manganese exposure can lead to accumulation in the rat striatum, the manganese content in the rat striatum was examined. The results indicated that manganese content in the LoMag group and the HiMag group was significantly higher than that in the Vehicle group $(\mathrm{P}<0.01)$. Manganese content in the HiMag + PBA group was similar to that in the HiMag group $(\mathrm{P}>0.05)$. Manganese content in the HiMag + PBA group was also significantly higher than that in the Vehicle group $(\mathrm{P}<0.01)$ (Table I). The results suggested that 4-PBA treatment did not reduce manganese content in the rat striatum exposed to a high dose of $\mathrm{MnCl}_{2}$.

4-PBA alleviates neuronal tissue damage caused by manganese exposure. In order to investigate whether manganese exposure causes damage to striatal neurons, H\&E staining 


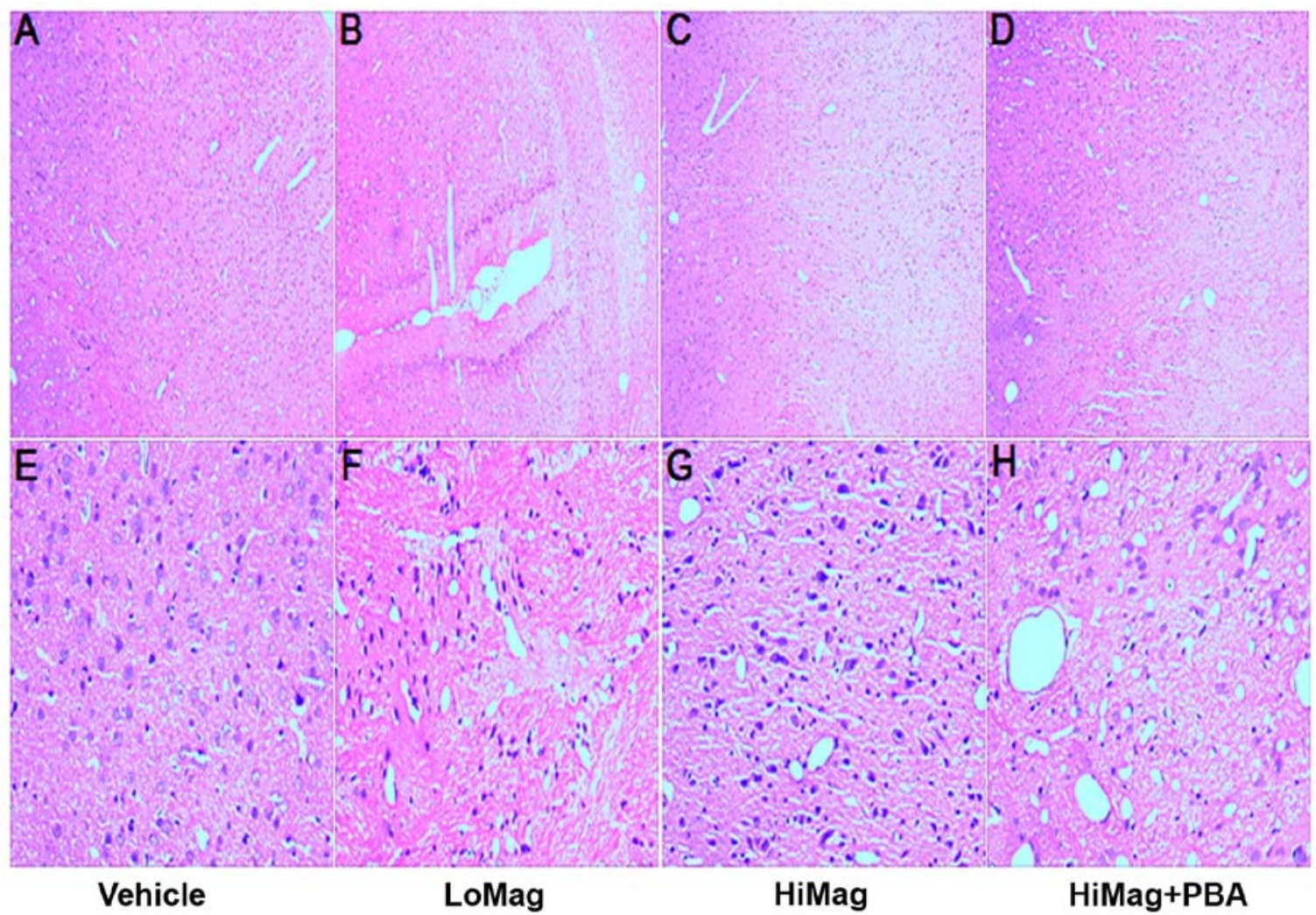

Figure 1. Hematoxylin and eosin staining analysis of rat striatum neurons in the four treatment groups. (A) Vehicle group, (B) LoMag group, (C) HiMag group, (D) HiMag + PBA group at magnification, x200. (E) Vehicle group (F) LoMag group, (G) HiMag group (H) HiMag + PBA group at magnification, x400. LoMag, $6 \mathrm{mg} / \mathrm{kg} \mathrm{MnCl}_{2}$; HiMag, $15 \mathrm{mg} / \mathrm{kg} \mathrm{MnCl}$; PBA, 4-phenylbutyrate.

Table I. Manganese content in the rat striatum of different groups.

\begin{tabular}{lccc}
\hline Group & Rats $(\mathrm{n})$ & $\begin{array}{c}\text { Manganese } \\
\text { content }(\mu \mathrm{g} / \mathrm{g})\end{array}$ & P-value $^{\mathrm{a}}$ \\
\hline Vehicle & 5 & $0.43 \pm 0.08$ & 1.00000 \\
LoMag & 5 & $1.02 \pm 0.11$ & 0.06000 \\
HiMag & 5 & $1.98 \pm 0.67$ & 0.00007 \\
HiMag+PBA & 5 & $1.93 \pm 0.62^{\mathrm{b}}$ & 0.00098 \\
\hline
\end{tabular}

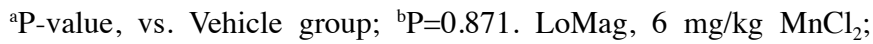
HiMag, $15 \mathrm{mg} / \mathrm{kg} \mathrm{MnCl}$; PBA, 4-phenylbutyrate.

analysis was performed (Fig. 1). In the Vehicle group, striatal neurons exhibited typical normal cellular features, including a clear, closely arranged structure, the nucleus in the middle of the cells, loose chromatin, nuclear membrane integrity and a clear nucleolus. However, neurons exhibited different morphologies in the manganese exposure groups, including disordered arrangement, ambiguous structure, a significantly swollen cytoplasm, volatile degeneration, eosinophilic formation, nuclear pyknosis and necrosis, and nuclear fragmentation or absence. In addition, the degree of damage was consistent with the dose of $\mathrm{MnCl}_{2}$. A high $\mathrm{MnCl}_{2}$ dose was closely associated with a higher level of damage. However, the damage was reduced by adding 4-PBA.
Table II. Apoptotic rate of rat striatal neurons in different treatment groups.

\begin{tabular}{lccr}
\hline Group & Rats (n) & Results & P-value $^{\mathrm{a}}$ \\
\hline Vehicle & 5 & $0.03 \pm 0.02$ & 1.00000 \\
LoMag & 5 & $0.16 \pm 0.01$ & $<0.00001$ \\
HiMag & 5 & $0.28 \pm 0.03$ & $<0.00001$ \\
HiMag+PBA & 5 & $0.14 \pm 0.02^{\mathrm{b}}$ & $<0.00001$ \\
\hline
\end{tabular}

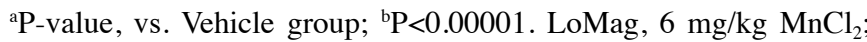
HiMag, 15 mg/kg $\mathrm{MnCl}_{2}$; PBA, 4-phenylbutyrate.

4-PBA reduces the number of apoptotic cells. In order to examine whether manganese exposure increases striatal neurons apoptosis, a TUNEL assay was performed to examine the striatum neuron apoptotic rates (Fig. 2 and Table II). TUNEL-positive cells were stained brown-yellow. In the Vehicle group, a small number of positive cells was observed (Fig. 2). However, the numbers of apoptotic cells in the manganese exposure groups were significantly higher than that in the Vehicle group (Table II). 4-PBA significantly reduced the number of positive cells $(\mathrm{P}<0.001$; Table II). However, the positive cell number in the HiMag + PBA group remained higher than that in the Vehicle group $(\mathrm{P}<0.01)$.

4-PBA helps to maintain normal cell ultrastructure. Electron microscopy revealed the ultrastructure changes in the 


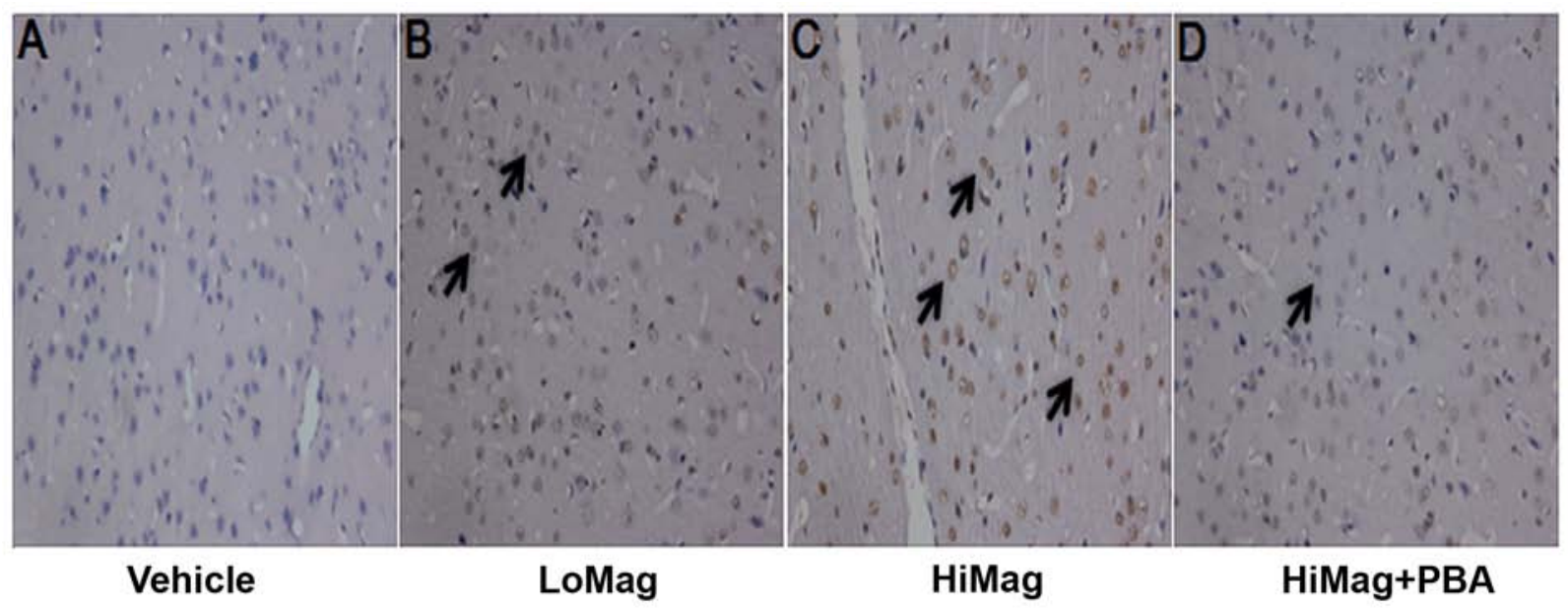

Figure 2.TUNEL assay of rat striatum neuron apoptosis in the four treatment groups. (A) Vehicle group,(B) LoMag group,(C) HiMag group and (D) HiMag + PBA group. Magnification, $\mathrm{x} 400$. The black arrows indicate apoptotic cells. LoMag, $6 \mathrm{mg} / \mathrm{kg} \mathrm{MnCl}{ }_{2} ; \mathrm{HiMag}, 15 \mathrm{mg} / \mathrm{kg} \mathrm{MnCl}$; PBA, 4-phenylbutyrate.

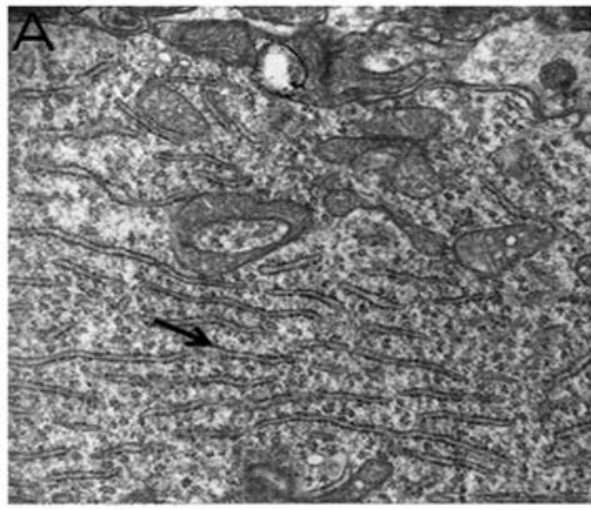

Vehicle

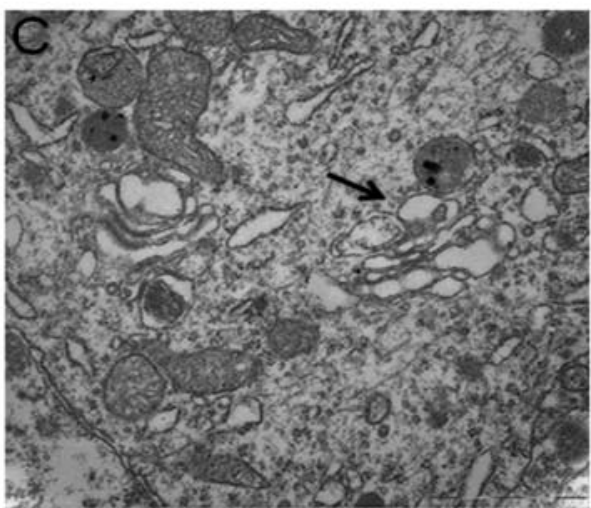

HiMag

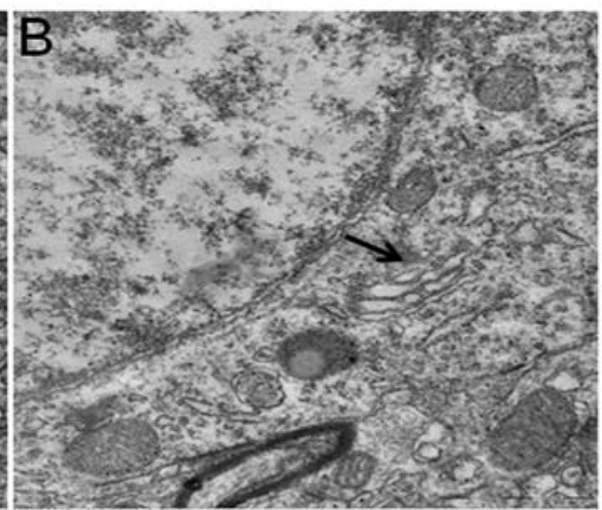

LoMag

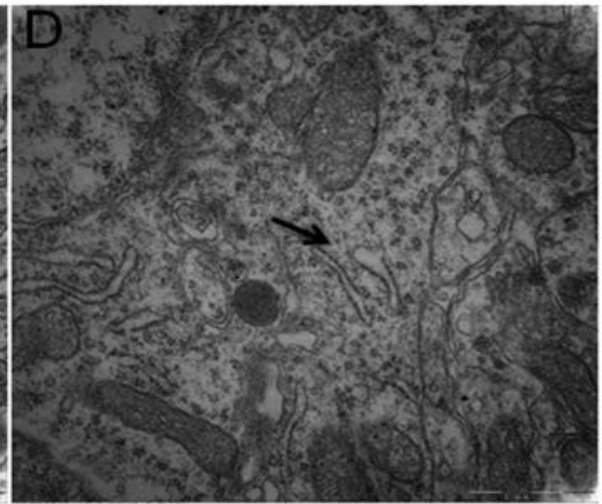

HiMag+PBA

Figure 3. Ultrastructure of striatal neurons examined by electron microscopy. ER structure in striatal neurons was examined in the (A) Vehicle group, (B) LoMag group, (C) HiMag group, (D) HiMag + PBA group. Black arrows indicate ER. Magnification, x40,000. LoMag, $6 \mathrm{mg} / \mathrm{kg} \mathrm{MnCl}$; HiMag, $15 \mathrm{mg} / \mathrm{kg}$ $\mathrm{MnCl}_{2} ; \mathrm{PBA}$, 4-phenylbutyrate; ER, endoplasmic reticulum.

differently treated groups (Fig. 3). In the Vehicle group, rat striatal neurons maintained a normal morphology, including loose nuclear chromatin, a clear nucleolus, and intact mitochondria, ER and other organelle structures. However, in the manganese exposure groups, cell morphology was significantly different. For example, nuclear chromatin condensation, swelling of ER, vacuolar formation, and ER and cell structure damage were observed. Following 4-PBA treatment, the damage effects were relieved (complete cell structure, and reduced degranulation and ER swelling).

4-PBA treatment reduces ERS-related protein expression. In order to identify the molecular changes between differently treated groups, western blot analysis was performed to determine the expression levels of GRP78, CHOP, JNK and caspase-12 (Figs. 4 and 5). The results suggested that the protein 


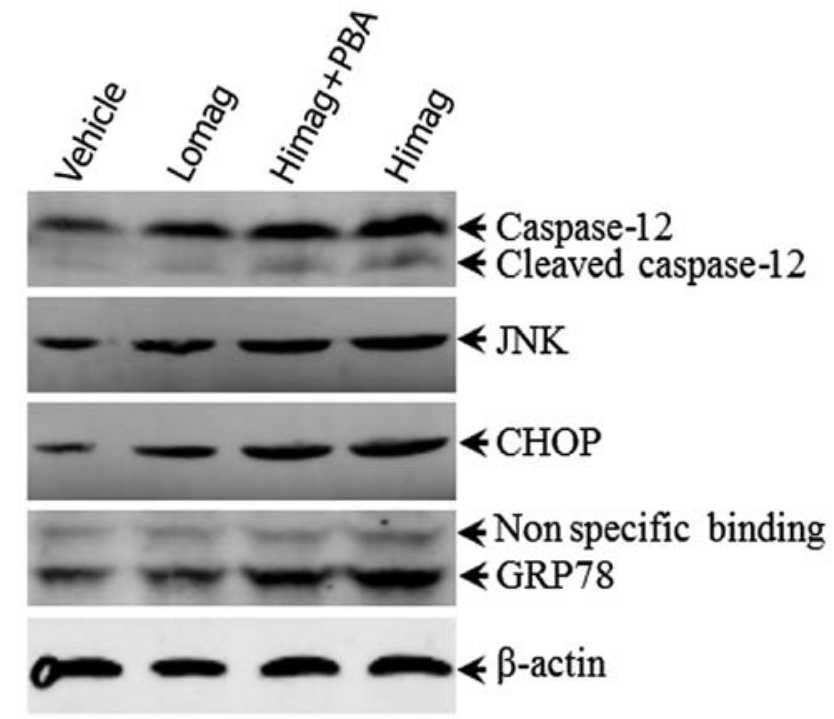

Figure 4. Western blot analyses of caspase-12, JNK, CHOP and GRP78 in the rat striatum. $\beta$-actin was used as an internal reference. Proteins expression was analyzed in the striatum of rats treated with LoMag, HiMag or HiMag + PBA. JNK, c-Jun N-terminal kinase; CHOP, C/EBP homologous protein; GRP78, glucose-regulated protein 78KD; LoMag, $6 \mathrm{mg} / \mathrm{kg} \mathrm{MnCl}_{2}$; HiMag, $15 \mathrm{mg} / \mathrm{kg} \mathrm{MnCl}$; PBA, 4-phenylbutyrate.

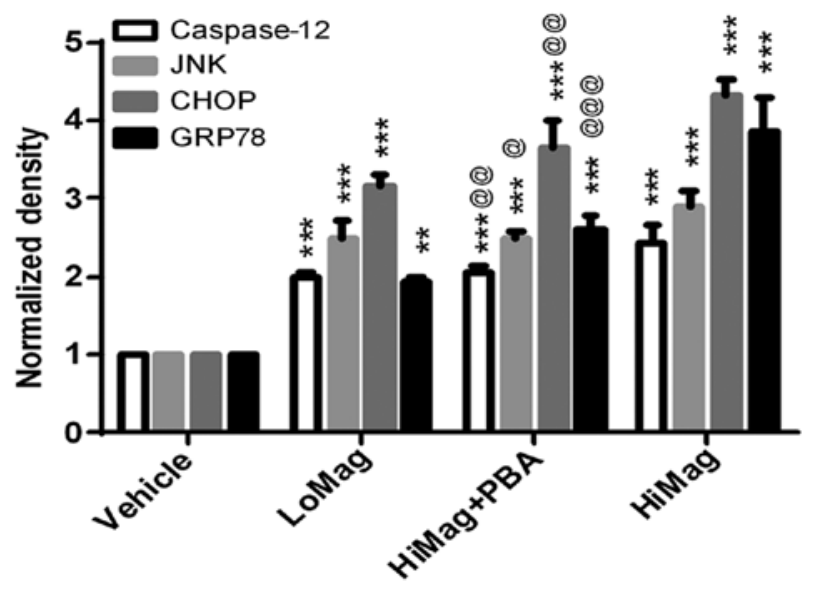

Figure 5. Quantitative protein expressions analysis of caspase-12, JNK, CHOP and GRP78 in the rat striatum. Proteins expression levels in the rat striatum were examined. The rat brains were collected from the LoMag group, HiMag group and HiMag + PBA group. ${ }^{* *} \mathrm{P}<0.01$ and ${ }^{* * *} \mathrm{P}<0.001$, compared with vehicle; ${ }^{\circledR} \mathrm{P}<0.05,{ }^{@} @ \mathrm{P}<0.01$ and ${ }^{\circledR @ @ ~} \mathrm{P}<0.001$, compared with HiMag. JNK, c-Jun N-terminal kinase; CHOP, C/EBP homologous protein; GRP78, glucose-regulated protein 78KD; LoMag, $6 \mathrm{mg} / \mathrm{kg} \mathrm{MnCl}{ }_{2}$; HiMag, $15 \mathrm{mg} / \mathrm{kg} \mathrm{MnCl} 2$;BA, 4-phenylbutyrate.

expression levels of GRP78, CHOP, JNK and caspase-12 in the LoMag and HiMag groups were significantly higher than those in the Vehicle group ( $\mathrm{P}<0.05$; Figs. 4 and 5$)$. The protein expression levels of GRP78, CHOP, JNK and caspase-12 in the HiMag + PBA group were significantly lower than those in the HiMag group $(\mathrm{P}<0.05)$. However, the expression levels of the four proteins in the HiMag + PBA group remained higher than those in the Vehicle group $(\mathrm{P}<0.05)$. These results indicated that 4-PBA effectively reduced ERS-related protein expression. However, 4-PBA did not fully eliminate the damage reduced by manganese exposure.

\section{Discussion}

The neurotoxicity mechanisms caused by manganese exposure include excitotoxicity, mitochondrial injury (19-21), ERS, oxygen free radical damage (22), thiol damage, dopamine auto-oxidation and iron metabolism (23). Wang et al (17) revealed that manganese can cause swelling of ER cell apoptosis and cell necrosis in rat neurons; whereas protein expression levels of ATF-6 $\alpha$, GRP78, PERK, CHOP, caspase-12 were upregulated and the expression of $\mathrm{Bcl}-2$ was downregulated. Xu et al (24) reported that manganese can induce ERS-induced apoptosis through PERK and IRE1 in the brain culture model. Seo et al (25) suggested that manganese exposure may damage cells via ERS responses and UPR responses. In addition, manganese can cause apoptosis through ER and mitochondrial dysfunction (26). ERS has dual functions for external stimuli. During initial stimulation, ERS is involved in adaptive regulation. The induction of ERS can reduce the harmful substances caused by renal damage in an in vitro cell culture model (27). Increased expression of GRP78 can reduce the apoptosis induced by ischemia (28). The upregulation of GRP78 can suppress the effects of harmful factors against hippocampal neurons (29). However, excessive or prolonged stress stimulation activates apoptotic signaling pathway-related proteins, including CHOP, JNK and caspase-12. This pathway leads to the removal of cells and structures that cannot be repaired. In addition, islet $\beta$-cell apoptosis induced by diabetes hyperglycemia, myocardial ischemia-reperfusion injury, nephron injury and renal failure are all associated with ERS (30-32).

In the present study, a sub-acute manganese exposure rat model was established by intraperitoneal injection with different doses of $\mathrm{MnCl}_{2}$ solution. The content of manganese in the striatum was detected. Compared with the Vehicle group, manganese levels in the LoMag group and HiMag groups were increased, which indicated that manganese can accumulate in the nervous system. H\&E staining revealed that striatal neurons in the $\mathrm{MnCl}_{2}$-exposed groups showed pyknosis, cell swelling and other structural damage. The TUNEL assay suggested that the number of apoptotic cells in the manganese-exposed groups was significantly increased. Electron microscopy revealed that the ER structure of the striatum was altered in the manganese-exposed groups. Swelling and degranulation appeared in the ER, and the degree of destruction in the ER was increased in a dose-dependent manner. Western blot analysis suggested that the protein expression levels of GRP78, CHOP, JNK and caspase-12 were also higher than those in the Vehicle group. These results indicated that $\mathrm{MnCl}_{2}$ exposure damaged the neurons of the striatum, ER structure and mitochondria. These adverse reactions promoted the expression of stress-related proteins, which led to the occurrence of apoptosis. ERS may be an important pathogenic mechanism for neurotoxicity caused by manganese exposure in rats.

The ERS inhibitor 4-PBA has been reported to assist abnormal proteins to regain the correct molecular structure in the ER $(33,34)$. A previous study showed that 4-PBA can effectively inhibit the activity of eIF-2 and PERK (11). 4-PBA has a protective role against ERS; therefore, 4-PBA reduced neuronal apoptosis in a cerebral ischemic injury mouse model (15). In addition, Mizukami et al (16) suggested that 4-PBA can inhibit ERS activation and improve motor impairment in rabbits. 
Currently, there is no relevant literature on the role of 4-PBA in inhibiting the ERS induced by manganese exposure. In the present study, 4-PBA did not reduce manganese content in the striatum of manganese-exposed rats. However, the results of H\&E staining and TUNEL assay revealed that 4-PBA improved the changes of cell structure and apoptotic cell number, which were caused by manganese exposure. The electron microscopy results suggested that 4-PBA reduced ER structure changes (swelling, vacuolization and degranulation). In addition, 4-PBA altered the protein expression levels of GRP78, CHOP, JNK and caspase-12 in the striatal neurons of rats exposed to a high dose of manganese. These experimental results suggested that 4-PBA reduced neuronal damage and apoptosis by inhibiting ERS.

In conclusion, the results of the present study demonstrated that ERS is key in striatal neuron apoptosis following sub-acute manganese exposure. 4-PBA improved multiple indicators; for example, 4-PBA reduced the expression of apoptosis-related proteins. These results provide a reliable reference for the early detection and treatment of manganese poisoning. However, there were limitations in the present study; for example, the experiment did not set different time points for manganese exposure. Therefore, further experiments with additional manganese dose treatments are required to identify ERS changes at different time points and different doses of manganese, to elaborate on these findings.

\section{Acknowledgements}

Not applicable.

\section{Funding}

This study was financially supported by the National Natural Science Foundation of China (grant no. 81460181) and the Natural Science Foundation of Guangxi, China (grant no. Junior program 2017JJB140225z).

\section{Availability of data and materials}

All data generated or analyzed during this study are included in this published article.

\section{Authors' contributions}

CW, GY and JW guaranteed the integrity of the present study, the clinical studies, data analysis and statistical analysis; JW and GY conceived and designed the study and the manuscript review; CW, RM and JW completed the study design; CW, GY, $\mathrm{RM}$ and JW revised the manuscript for important intellectual content; CW, GY and JW performed the literature research; CW, RM, YH, GY and TL performed the experimental studies and data acquisition; $\mathrm{CW}, \mathrm{YH}$ and TL were involved in manuscript preparation; CW, GY and JW edited the manuscript. All authors read and approved the final manuscript.

\section{Ethics approval and consent to participate}

The experiments were approved by the Committee on the Ethics of Animal Experiments of the First Affiliated Hospital of Guangxi Medical University [permit no. 2015 (KY-E-67)].

\section{Patient consent for publication}

Not applicable.

\section{Competing interests}

The authors declare that they have no competing interests.

\section{References}

1. Berger MM and Shenkin A: Vitamins and trace elements: Practical aspects of supplementation. Nutrition 22: 952-955, 2006.

2. Nutt DJ, Baldwin DS, Clayton AH, Elgie R, Lecrubier Y, Montejo AL, Papakostas GI, Souery D, Trivedi MH and Tylee A: Consensus statement and research needs: The role of dopamine and norepinephrine in depression and antidepressant treatment. J Clin Psychiatry 67 (Suppl 6): S46-S49, 2006.

3. Moussavi S, Chatterji S, Verdes E, Tandon A, Patel V and Ustun B: Depression, chronic diseases, and decrements in health: Results from the world health surveys. Lancet 370: 851-858, 2007.

4. Burton NC and Guilarte TR: Manganese neurotoxicity: Lessons learned from longitudinal studies in nonhuman primates. Environ Health Perspect 117: 325-332, 2009.

5. Sundar Rajan S, Srinivasan V, Balasubramanyam M and Tatu U: Endoplasmic reticulum (ER) stress \& diabetes. Indian J Med Res 125: 411-424, 2007.

6. Mu YP, Ogawa T and Kawada N: Reversibility of fibrosis, inflammation, and endoplasmic reticulum stress in the liver of rats fed a methionine-choline-deficient diet. Lab Invest 90: 245-256, 2010.

7. McCullough KD, Martindale JL, Klotz LO, Aw TY and Holbrook NJ: Gadd153 sensitizes cells to endoplasmic reticulum stress by down-regulating $\mathrm{Bcl} 2$ and perturbing the cellular redox state. Mol Cell Biol 21: 1249-1259, 2001.

8. Oyadomari S and Mori M: Roles of CHOP/GADD153 in endoplasmic reticulum stress. Cell Death Differ 11: 381-389, 2004.

9. Nishitoh H, Matsuzawa A, Tobiume K, Saegusa K, Takeda K, Inoue $\mathrm{K}$, Hori S, Kakizuka A and Ichijo H: ASK1 is essential for endoplasmic reticulum stress-induced neuronal cell death triggered by expanded polyglutamine repeats. Genes Dev 16: 1345-1355, 2002.

10. Yoneda T, Imaizumi K, Oono K, Yui D, Gomi F, Katayama T and Tohyama M: Activation of caspase-12, an endoplastic reticulum (ER) resident caspase, through tumor necrosis factor receptor-associated factor 2-dependent mechanism in response to the ER stress. J Biol Chem 276: 13935-13940, 2001.

11. Ozcan U, Yilmaz E, Ozcan L, Furuhashi M, Vaillancourt E, Smith RO, Görgün CZ and Hotamisligil GS: Chemical chaperones reduce ER stress and restore glucose homeostasis in a mouse model of type 2 diabetes. Science 313: 1137-1140, 2006.

12. Choi SE, Lee YJ, Jang HJ, Lee KW, Kim YS, Jun HS, Kang SS, Chun J and Kang Y: A chemical chaperone 4-PBA ameliorates palmitate-induced inhibition of glucose-stimulated insulin secretion (GSIS). Arch Biochem Biophys 475: 109-114, 2008.

13. Luo ZF, Feng B, Mu J, Qi W, Zeng W, Guo YH, Pang Q, Ye ZL, Liu L and Yuan FH: Effects of 4-phenylbutyric acid on the process and development of diabetic nephropathy induced in rats by streptozotocin: Regulation of endoplasmic reticulum stress-oxidative activation. Toxicol Appl Pharmacol 246: 49-57, 2010.

14. Vilatoba M, Eckstein C, Bilbao G, Smyth CA, Jenkins S, Thompson JA, Eckhoff DE and Contreras JL: Sodium 4-phenylbutyrate protects against liver ischemia reperfusion injury by inhibition of endoplasmic reticulum-stress mediated apoptosis. Surgery 138: 342-351, 2005.

15. Qi X, Hosoi T, Okuma Y, Kaneko M and Nomura Y: Sodium 4-phenylbutyrate protects against cerebral ischemic injury. Mol Pharmacol 66: 899-908, 2004.

16. Mizukami T, Orihashi K, Herlambang B, Takahashi S, Hamaishi M, Okada K and Sueda T: Sodium 4-phenylbutyrate protects against spinal cord ischemia by inhibition of endoplasmic reticulum stress. J Vasc Surg 52: 1580-1586, 2010.

17. Wang T, Li X, Yang D, Zhang H, Zhao P, Fu J, Yao B and Zhou Z: ER stress and ER stress-mediated apoptosis are involved in manganese-induced neurotoxicity in the rat striatum in vivo. Neurotoxicology 48: 109-119, 2015. 
18. Silva DN, Coelho J, Frazílio Fde O, Odashiro AN, Carvalho Pde T, Pontes ER, Vargas AF, Rosseto M and Silva AB: End-to-side nerve repair using fibrin glue in rats. Acta Cir Bras 25: 158-162, 2010.

19. Zhang S, Fu J and Zhou Z: Changes in the brain mitochondrial proteome of male Sprague-Dawley rats treated with manganese chloride. Toxicol Appl Pharmacol 202: 13-17, 2005.

20. Dobson AW, Erikson KM and Aschner M: Manganese neurotoxicity. Ann N Y Acad Sci 1012: 115-128, 2004.

21. Zhang S, Zhou Z and Fu J: Effect of manganese chloride exposure on liver and brain mitochondria function in rats. Environ Res 93: 149-157, 2003.

22. Zhang S, Fu J and Zhou Z: In vitro effect of manganese chloride exposure on reactive oxygen species generation and respiratory chain complexes activities of mitochondria isolated from rat brain. Toxicol In Vitro 18: 71-77, 2004.

23. Pang L, Wang J, Huang W and Guo S: A study of divalent metal transporter 1 and ferroportin 1 in brain of rats with manganese-induced parkinsonism. Zhonghua Lao Dong Wei Sheng Zhi Ye Bing Za Zhi 33: 250-254, 2015 (In Chinese).

24. Xu B, Shan M, Wang F, Deng Y, Liu W, Feng S, Yang TY and $\mathrm{Xu}$ ZF: Endoplasmic reticulum stress signaling involvement in manganese-induced nerve cell damage in organotypic brain slice cultures. Toxicol Lett 222: 239-246, 2013.

25. Seo YA, Li Y and Wessling-Resnick M: Iron depletion increases manganese uptake and potentiates apoptosis through ER stress. Neurotoxicology 38: 67-73, 2013.

26. Yoon H, Kim DS, Lee GH, Kim KW, Kim HR and Chae HJ: Apoptosis induced by manganese on neuronal SK-N-MC Cell Line: Endoplasmic reticulum (ER) stress and mitochondria dysfunction. Environ Health Toxicol 26: e2011017, 2011.

27. Peyrou M and Cribb AE: Effect of endoplasmic reticulum stress preconditioning on cytotoxicity of clinically relevant nephrotoxins in renal cell lines. Toxicol In Vitro 21: 878-886, 2007.
28. Wu X, Zhao H, Min L, Zhang C, Liu P and Luo Y: Effects of 2-Deoxyglucose on ischemic brain injuries in rats. Int $\mathrm{J}$ Neurosci 124: 666-672, 2014

29. Yu Z, Luo H, Fu W and Mattson MP: The endoplasmic reticulum stress-responsive protein GRP78 protects neurons against excitotoxicity and apoptosis: Suppression of oxidative stress and stabilization of calcium homeostasis. Exp Neurol 155: 302-314, 1999.

30. Oyadomari S, Araki E and Mori M: Endoplasmic reticulum stress-mediated apoptosis in pancreatic beta-cells. Apoptosis 7: 335-345, 2002.

31. Kim DS, Kwon DY, Kim MS, Kim HK, Lee YC, Park SJ, Yoo WH, Chae SW, Chung MJ, Kim HR and Chae HJ: The involvement of endoplasmic reticulum stress in flavonoid-induced protection on cardiac cell death caused by ischaemia/reperfusion. J Pharm Pharmacol 62: 197-204, 2010.

32. Nakajo A, Khoshnoodi J, Takenaka H, Hagiwara E, Watanabe T, Kawakami H, Kurayama R, Sekine Y, Bessho F, Takahashi S, et al: Mizoribine corrects defective nephrin biogenesis by restoring intracellular energy balance. J Am Soc Nephrol 18: 2554-2564, 2007.

33. Malo A, Krüger B, Göke B and Kubisch CH: 4-Phenylbutyric acid reduces endoplasmic reticulum stress, trypsin activation, and acinar cell apoptosis while increasing secretion in rat pancreatic acini. Pancreas 42: 92-101, 2013.

34. Mimori S, Okuma Y, Kaneko M, Kawada K, Hosoi T, Ozawa K, Nomura Y and Hamana H: Protective effects of 4-phenylbutyrate derivatives on the neuronal cell death and endoplasmic reticulum stress. Biol Pharm Bull 35: 84-90, 2012. 